\title{
SPECTRA OF CONSERVATIVE MATRICES
}

\author{
N. K. SHARMA
}

ABSTRACT. In this paper we study the spectra of conservative matrices and show that the spectrum of any Hausdorff method is either uncountable or finite. In the latter case it is shown that the spectrum consists of either one point or two points. We obtain the sharpest possible Mercerian theorem for Euler methods. We also get some results about the location of conservative matrices with respect to the maximal group of invertible operators.

Let $\omega$ represent the space of all sequences and let $c\left(c_{0}\right.$ or $\left.m\right)$ represent the space of convergent sequences (null sequences or bounded sequences). Let $B(c)$ represent the Banach algebra of bounded operators on $c$ with the usual norm topology. Let $A=\left(a_{n k}\right)$ be any infinite matrix and let, for an $x$ in $\omega, A x$ denote the transformed sequence, provided it exists. Let $c_{A}=\{x \in \omega: A x \in c\} . A$ is called conservative (coercive) if $c_{A} \supseteq c\left(c_{A} \supseteq m\right)$. For the properties of conservative (coercive) matrices and Hausdorff methods the reader might refer to [1].

In this paper we first obtain information about the spectrum of a conservative Hausdorff method and use this information to prove that a Hausdorff method is coercive if and only if it is a scalar multiple of $H_{0}$ which we will define later on. This generalizes the result about right finite Hausdorff methods which appears in [3]. Incidentally we show that the answers to Questions 1 and 4(i) of Rhoades [4] are in the affirmative. Then we discuss the spectral aspects of Mercer's theorem for Euler's methods. These results show that the discussion about the location of Euler's methods in [4] is not correct. Finally we list two open problems about the structure of the spectra of conservative matrices. We wish to thank Professor B. E. Rhoades for many fruitful conversations.

A matrix $A$ is called triangular if $a_{n k}=0$ whenever $k>n$ and a triangular matrix $A$ is called a triangle if $a_{n n} \neq 0$ for any $n$. $\Delta$ denotes the Banach algebra of triangular matrices in $B[c], G$ denotes the group of invertible operators in $\Delta$ and $\partial G$ the boundary of $G$ in $\Delta$. The following theorem about coercive triangular matrices has been essentially proved in [5].

Presented to the Society, January 17, 1972; received by the editors November 18, 1971 and, in revised form, February 24, 1972.

AMS 1970 subject classifications. Primary 40H05, 40G05; Secondary 47B99.

c American Mathematical Society 1972 
THEOREM 1. Let $A=\left(a_{n k}\right)$ be a coercive triangular matrix. Then $\sigma(A)=\left\{0, a_{11}, a_{22}, a_{33}, \cdots\right\}$.

One immediate consequence of Theorem 1 is the well-known fact that every coercive matrix is in $\partial G$.

The second consequence is an affirmative answer to Question 1 of Rhoades [4], namely the existence of triangles $A \notin G$, and $B \in \partial G$ with $B A \in \partial G$. To see this we take $B$ to be an arbitrary coercive triangle. Then for any triangle $A, B A$ is coercive. Similarly we answer Question 4(i) by exhibiting a triangle not of type $M$ on $\partial G$. Let $A$ be any triangle which is not of type $M, B$ any coercive triangle. Then $A B$ is the required example.

For studying the structure of the spectrum of a conservative Hausdorff method $H=\left(h_{n k}\right)$ we need the following

FACT 1. Corresponding to a conservative Hausdorff method $H$, there exists $\mu$ analytic in the right half-plane such that $h_{n n}=\mu(n)$ for $n=0$, $1,2,3, \cdots$.

FACr 2 [3]. If $M$ denotes the Cesàro method of order 1 and $f$ is any function of a complex variable, then $f(M)$ denotes a matrix $\left(f_{n k}\right)$ where

$$
f_{n k}=\sum_{h=k}^{n}(-1)^{h-k} \frac{(n-1) !}{(n-h) !(h-k) !(k-1) !} f\left(\frac{1}{h}\right)
$$

which commutes with $M$.

If we take $f(z)=\mu(1 / z-1)$ then $f(M)=H$. Let $D=\left\{z:\left|z-\frac{1}{2}\right|<\frac{1}{2}\right\}$.

THEOREM 2. The spectrum of a conservative Hausdorff method is either finite or uncountably infinite. In case it is finite it consists of either one point or two points.

Proof. Let $H$ be any conservative Hausdorff method. Let $f$ be the function corresponding to $H$ as given by Fact 2. Then $f$ is analytic in $D$ and $f(M)=H$. If $\alpha$ is in $D$, then $1 /(f(z)-f(\alpha))$ is not analytic in $D$. Consequently $f(M)-f(\alpha) I$ is not invertible and therefore $\sigma(H) \supset f(D)$. In case $f$ is not a constant in $D$, in view of the local mapping theorem for analytic functions we get that $\sigma(H)$ contains an open set and therefore $\sigma(H)$ is uncountable. On the other hand if $f$ is a constant in $D$ then

$$
H=\left(\begin{array}{cccc}
\beta & 0 & 0 & \cdots \\
\beta-\alpha & \alpha & 0 & \cdots \\
\beta-\alpha & 0 & \alpha & \cdots \\
& \ldots & &
\end{array}\right)
$$

where $\alpha$ and $\beta$ are any two complex numbers. Clearly $\sigma(H)=\{\alpha, \beta\}$ and the theorem is established. 
Corollary 1. A Hausdorff method $H$ is coercive if and only if $H=$ $\beta H_{0}$ where $\beta$ is a scalar and

$$
H_{0}=\left(\begin{array}{ll}
1 & \\
1 & \\
1 & 0 \\
\cdot & \\
\cdot &
\end{array}\right)
$$

Proof. Since $H$ is coercive, by Theorem $1, \sigma(H)$ is countable. Hence from Theorem 2, the diagonal of $H=\{\beta, \alpha, \alpha, \alpha, \cdots\}$. It is well known that the diagonal of a coercive matrix is a null sequence. Thus we must have $\alpha=0$. It follows from (*) that $H=\beta H_{0}$. The converse is trivial.

Since a right finite matrix is necessarily coercive, the result about right finite Hausdorff matrices that appears at the bottom of p. 10 in [3] is immediate.

Mercer in 1906 proved that, if $M$ denotes the Cesàro matrix of order one, then the convergence domain of $\alpha I+(1-\alpha) M$ for $\alpha>0$ equals $c$.

Clearly Mercer's theorem is a statement about the spectrum of the Cesàro matrix. Indeed the result of [2] that this spectrum is $\left\{z:\left|z-\frac{1}{2}\right| \leqq \frac{1}{2}\right\}$ is equivalent to Hardy's result $[1, p .106]$ that $\alpha I+(1-\alpha) M$ is Mercerian iff $\operatorname{Re} \alpha>0$. This equivalence yields a Mercerian theorem for certain Hausdorff methods proved below, but first we need the following lemma because for any conservative matrix $A, 0 \in \sigma(A)$ does not necessarily mean that $c_{A} \supsetneq c$.

Lemma. Let $A$ be a triangular matrix with at most a finite number of zeros on the main diagonal. If $c_{A}=c$, then 0 is an isolated point of $\sigma(A)$.

Proof. Let $A^{\prime}$ be the matrix obtained from $A$ by replacing zeros of $A$ on the main diagonal with any number $\varepsilon \neq 0$. Clearly $c_{A^{\prime}}=c_{A^{\prime}}$. Since $A^{\prime}$ is a triangle, $0 \notin \sigma\left(A^{\prime}\right)$. Observe that $\sigma(A)$ and $\sigma\left(A^{\prime}\right)$ differ only by 0 and $\varepsilon$. This proves the lemma.

THEOREM 3. Let $E$ be the Hausdorff method corresponding to the sequence $\left\{1, q, q^{2}, \cdots\right\}, 0<q<1$. Then $\alpha I+(1-\alpha) E$ is Mercerian if and only if $|\alpha|>|1-\alpha|$. Thus if $\alpha>0, \alpha I+(1-\alpha) E$ is Mercerian if and only if $\alpha>\frac{1}{2}$.

REMARK. The last statement of Theorem 3 is a well-known result.

Proof. The analytic function $f$ corresponding to $E$ is $g^{1 / z-1}$. Observe that $z \rightarrow q^{1 / z-1}$ maps $\left\{z:\left|z-\frac{1}{2}\right|<\frac{1}{2}\right\}$ onto $\{z: 0<|z|<1\}$. Hence from Theorem 2, $\sigma(E) \supset\{z: 0<|z|<1\}$. Since the spectrum is closed, we get $\sigma(E) \supseteq$ $\{z:|z| \leqq 1\}$. Observe that the Banach norm of $E$ is 1 to conclude that $\sigma(E)=\{z:|z| \leqq 1\}$. The theorem now follows because of the lemma above. 
Open problems. It is well known that given any compact subset $S$ of the complex plane, there exists a matrix $A$ which is a bounded operator on $c_{0}($ or $m)$ such that $\sigma(A)=S$.

Problem 1. Does there exist a conservative matrix $A$ such that $\sigma(A)=S$ ? The second problem is about the converse of the lemma.

Problem 2. Is there a conservative triangle with 0 as an isolated point of the spectrum?

We are grateful to the referee of this paper for his many valuable remarks.

\section{REFERENCES}

1. G. H. Hardy, Divergent series, Clarendon Press, Oxford, 1949. MR 11, 25.

2. W. A. Hurwitz and L. L. Silverman, On the consistency and equivalence of certain definitions of summability, Trans. Amer. Math. Soc. 18 (1917), 1-20.

3. B. E. Rhoades, Some structural properties of Hausdorff matrices, Bull. Amer. Math. Soc. 65 (1959), 9-11. MR 20 \#7171.

4. - Triangular summability methods and the boundary of the maximal group, Math. Z. 105 (1968), 284-290. MR 37 \#4461.

5. A. Wilansky and $\mathrm{K}$. Zeller, Banach algebra and summability, Illinois J. Math. 2 (1958), 378-385; erratum, ibid. 3 (1959), 468.

Department of Mathematics, Indiana Universitr, Bloomington, Indiana 47401

Current address: Department of Mathematics, Pahlavi University, Shiraz, Iran 